\title{
Monocyte and macrophage differentiation: circulation inflammatory monocyte as biomarker for inflammatory diseases
}

\author{
Jiyeon Yang, Lixiao Zhang, Caijia Yu, Xiao-Feng Yang and Hong Wang*
}

\begin{abstract}
Monocytes express various receptors, which monitor and sense environmental changes. Monocytes are highly plastic and heterogeneous, and change their functional phenotype in response to environmental stimulation. Evidence from murine and human studies has suggested that monocytosis can be an indicator of various inflammatory diseases. Monocytes can differentiate into inflammatory or anti-inflammatory subsets. Upon tissue damage or infection, monocytes are rapidly recruited to the tissue, where they can differentiate into tissue macrophages or dendritic cells. Given the rapid progress in monocyte research from broad spectrum of inflammatory diseases, there is a need to summarize our knowledge in monocyte heterogeneity and its impact in human disease. In this review, we describe the current understanding of heterogeneity of human and murine monocytes, the function of distinct subsets of monocytes, and a potential mechanism for monocyte differentiation. We emphasize that inflammatory monocyte subsets are valuable biomarkers for inflammatory diseases, including cardiovascular diseases.
\end{abstract}

Keywords: Monocytes, Inflammatory diseases

\section{Introduction}

The primary role of monocytes was considered to sense the environment and replenish the pool of tissue macrophages and dendritic cells. Recent advances in immunology research have discovered that monocytes are heterogenic and can be divided into three subsets based on specific surface markers and that each subset displays specific functions. During steady state, circulating monocytes have a half-life of about one to three days, and maintain a steady composition of monocyte subsets.

Identified monocyte subsets exhibit distinct pathophysiological roles. Classical inflammatory monocytes are equipped with a set of Toll-like receptors (TLRs) and scavenger receptors, recognizing pathogen-associated molecular patterns (PAMPs) and removing microorganisms, lipids, and dying cells via phagocytosis. They produce effector molecules such as cytokines, myeloperoxidase and superoxide, and initiate inflammation [1].

\footnotetext{
* Correspondence: hongw@temple.edu

Department of Pharmacology, Centers for Metabolic Disease Research,

Cardiovascular Research, and Thrombosis Research, Temple University School of Medicine, Philadelphia, PA 19140, USA
}

Inflammatory monocytes selectively traffic to the sites of inflammation, produce inflammatory cytokines and contribute to local and systemic inflammation [2]. They are highly infiltrative and can be differentiated into inflammatory macrophages, which remove PAMPs and cell debris. In steady state, the patrolling anti-inflammatory monocytes patrol the vasculature to monitor PAMPs and become tissue resident macrophages. During inflammation, they differentiate into anti-inflammatory macrophages, which repair damaged tissues [3].

Murine monocyte subset classification and their functional determinations have been consistent and well accepted [4]. However, classification of human monocyte subsets in relevance to their inflammatory or antiinflammatory functional phenotypes remains partially undefined. Here, we intend to review the current understanding regarding monocyte heterogeneity, and to integrate the knowledge of murine and human monocyte classification.

\section{Monocytosis and heterogeneous monocytes}

It was first reported in the 1970s that monocytes increase proliferative activity in bone marrow (BM) in 
response to inflammatory stimuli, leading to monocytosis, [5] a clinical condition reflecting an increased number of circulating monocytes.

Emerging clinical analysis revealed a higher prevalence of monocytosis in cardiovascular diseases (CVD) (Table 1). Monocyte count is increased in acute myocardial infarction (AMI) patients compared to patients with stable coronary arterial disease (CAD) [6]. Peripheral monocytosis is associated with left ventricular (LV) dysfunction and LV aneurysm, suggesting a possible role of monocytes in the development of LV remodeling after reperfused AMI [7]. Monocytosis is also associated with reduced high-density lipoprotein (HDL) levels and impaired renal function in CAD patients [8]. It has been demonstrated that monocyte count is a better independent risk factor of CVD than several conventional risk factors such as C-reactive protein (CRP), inflammatory cytokine interleukin-6 (IL-6), fibrinogen, hypertension, and cigarette smoking [9]. The treatment of coronary arterial disease patients with pravastatin, a cholesterol lowering medication, for 6 months reduces plaque volume and monocyte count, implying that monocytosis is a potential target for coronary atherosclerotic regression [10].

Following the defining of monocytosis, reduced phagocytic capacity of monocytes was found in patients with rheumatoid arthritis and cutaneous vasculitis [11]. Patients with lymphopenia have suppressor monocytes, which are unable to activate T-cells [12]. These findings suggested the existence of heterogeneous monocyte populations. Further studies for different functional properties of such populations identified that CD16 (Leu-11), a Fc receptor (FcR) as it binds to the Fc region (constant region) of antibody, is expressed on the surface of monocytes and correlated with atherosclerosis and CVD in patients and an inflammatory phenotype in cultured monocytes and circulating monocytes [13]. The CD16 monocytes has been considered an inflammatory monocyte subset in humans [14].

\section{Mouse monocyte subsets}

Monocyte subsets in mice were first identified by differential expression of chemokine receptors CCR2. CCR2 ${ }^{+}$subset shows higher migratory and infiltration capacity than CCR2 ${ }^{-}$subset and was initially considered as murine inflammatory monocyte [15]. Later on, mouse monocyte subsets are characterized by differential expression of an inflammatory monocyte marker Ly6C (Gr1). It is now accepted that mouse monocyte subsets are grouped as

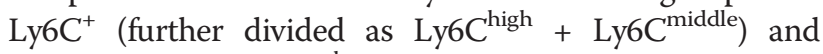
Ly6C (also called Ly6C ${ }^{\text {low }}$ ) monocyte subsets based on expression levels of Ly6C on cell surface (Table 2). The surface markers and chemokine receptors for $\mathrm{Ly}_{6 \mathrm{C}}{ }^{+}$subsets are $\mathrm{CD}_{11} \mathrm{~b}^{+} \mathrm{CD} 115^{+}$and $\mathrm{CCR} 2^{\text {high }} \mathrm{CX} 3 \mathrm{CR} 1^{\text {low }}$. Whereas, the surface markers and chemokine receptors for Ly $6 \mathrm{C}^{-}$monocytes are $\mathrm{CD} 11 \mathrm{~b}^{+} \mathrm{CD} 115^{+}$and $\mathrm{CCR} 2{ }^{\text {low }} \mathrm{CX} 3 \mathrm{CR} 1^{\text {high }}[16]$.

\section{Functional properties of mouse monocyte subsets}

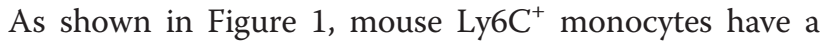
high antimicrobial capability due to their potent capacity for phagocytosis, secrete ROS, TNF $\alpha$, nitric oxide, IL-1 $\beta$, little IL-10 upon bacterial infection [17] and large amount of type 1 interferon (IFN) in response to viral ligands [18]. CCR2-CCL2 signaling in $\mathrm{Ly}^{+} \mathrm{C}^{+}$monocytes alters the conformational change of VLA-4 ( $\alpha 4 \beta 1$ integrin), the ligand for VCAM-1, leading to high affinity interaction and monocyte transmigration (Figure 1). In vascular inflammation, Ly6 $\mathrm{C}^{+}$monocytes are preferentially recruited into inflamed tissue via interaction of chemokine receptor CCR2 [19] and more likely to mature to inflammatory M1 macrophages, which are distinguished by secretion of pro-inflammatory cytokine, TNF $\alpha$, and IL- 6 and contribute to tissue degradation and $\mathrm{T}$ cell activation.

In steady state, $\mathrm{Ly} 6 \mathrm{C}^{+}$monocytes differentiate into Ly $6 \mathrm{C}^{-}$monocytes in the circulation. This subset patrols the luminal side of endothelium of small blood vessels and bind to endothelium by chemokine receptor

Table 1 Monocytosis in human disease

\begin{tabular}{|c|c|c|c|}
\hline Disease & Group comparison & Monocyte counts comparison & PMID \# \\
\hline$\overline{C V D}$ & $\mathrm{HDL}, 2 \mathrm{nmol} / \mathrm{L}$ vs. $<1 \mathrm{nmol} / \mathrm{L}$ & $3.65 \times 10^{8}$ vs. $4.5 \times 10^{8}$ cells $/ \mathrm{dL}$ & 18629357 \\
\hline AMl & CT vs. AMl & $4.97 \times 10^{8}$ vs. $7.93 \times 10^{8}$ cells $/ \mathrm{dL}$ & 23455782 \\
\hline \multirow[t]{3}{*}{ AMl } & 5 h AMl vs. $1-2$ day AMl & $4.56 \times 10^{8}$ vs. $7.11 \times 10^{8}$ cells $/ \mathrm{dL}$ & 11788214 \\
\hline & None vs. Pump failure & $6.05 \times 10^{8}$ vs. $9.41 \times 10^{8}$ cells $/ \mathrm{dL}$ & \\
\hline & None vs. LV aneurysm & $6.82 \times 10^{8}$ vs. $8.61 \times 10^{8}$ cells $/ \mathrm{dL}$ & \\
\hline CKD & CKD without CVD vs. CKD with CVD & $5.71 \times 10^{8}$ vs. $6.97 \times 10^{8}$ cells $/ \mathrm{dL}$ & 18160960 \\
\hline AMl & LVF recovery vs. LVF no recovery & $6.42 \times 10^{8}$ vs. $10.13 \times 10^{8}$ cells $/ \mathrm{dL}$ & 17652884 \\
\hline$C A D$ & Healthy vs. CAD vs. AMI & $5.17 \times 10^{8}$ vs. $5.42 \times 10^{8}$ vs. $6.72 \times 10^{8}$ cells $/ \mathrm{dL}$ & 16612453 \\
\hline
\end{tabular}

Circulating total MC counts were examined in human disease as indicated. Comparisons were made between described groups in CVD, and MC counts. We used PMID \# to cite individual manuscripts reporting these studies. $A M I$, acute myocardial infarction; $C A D$, coronary arterial disease; $C K D$, chronic kidney disease; $C K$, creatine kinase; CRP, C-reactive protein; CVD, cardiovascular disease; LVF, left ventricular failure. PMID, PubMed Identification. 
Table 2 Markers and functions of MC subsets in human and mouse

\begin{tabular}{|c|c|c|c|c|c|}
\hline Species & Subsets & Surface markers & $\%$ in $\mathrm{MNC}$ & Chemokine receptors & Functions \\
\hline \multirow[t]{3}{*}{ Human } & Classical & $\mathrm{CD} 14^{++} \mathrm{CD} 16^{-}$ & $80-95$ & $\mathrm{CCR} 2{ }^{\text {high }} \mathrm{CX} 3 \mathrm{CR} 1^{\text {low }}$ & Phagocytosis \\
\hline & Intermediate & $\mathrm{CD} 14^{++} \mathrm{CD} 16^{+}$ & $2-11$ & $\mathrm{CCR}^{\text {mid }} \mathrm{CX} 3 \mathrm{CR} 1^{\text {high }} \mathrm{CCR}^{+}$ & Pro-inflammatory \\
\hline & Non-classical & $\mathrm{CD} 14^{+} \mathrm{CD} 16^{++}$ & $2-8$ & $C C R 2^{\text {low }} C X 3 C R 1^{\text {high }}$ & Patrolling \\
\hline \multirow[t]{3}{*}{ Mouse } & Ly6C high $\left(\right.$ Ly6C $\left.^{+}\right)$ & CD $11 b^{+} \mathrm{CD} 115^{+}$Ly6C high & $40-45$ & $\mathrm{CCR} 22^{\text {high }} \mathrm{C} \times 3 \mathrm{CR} 1^{\text {low }}$ & Phagocytosis \& Pro-inflammatory \\
\hline & Ly $6 C^{\text {middle }}\left({\text { Ly } 6 C^{+}}^{+}\right)$ & CD11 b ${ }^{+}$CD $115^{+}{\text {Ly } 6 C^{\text {middle }}}$ & $5-32$ & $\mathrm{CCR} 2^{\text {high }} \mathrm{CX} 3 \mathrm{CR} 1^{\text {low }}$ & Pro-inflammatory \\
\hline & Ly6C low $^{\text {Ly6C }}$ ) & CD11b $b^{+} \mathrm{CD} 115^{+}$Ly6C $^{\text {low }}$ & $26-50$ & $\mathrm{CCR} 2^{\text {low }} \mathrm{C} \times 3 \mathrm{CR} 1^{\text {high }}$ & Patrolling; tissue repair \\
\hline
\end{tabular}

Human MCs are divided into three subsets based on the cell surface expression of CD14 and CD16. CD14 ${ }^{++}$CD16 ${ }^{-}$MCs, also called the classical MC, are the most prevalent MC subset in human blood and express high level of CCR2. The CD14 ${ }^{++} \mathrm{CD} 16^{+} \mathrm{MCs}$ are intermediate MC which contribute significantly to atherosclerosis. The $\mathrm{CD} 14^{+} \mathrm{CD} 16^{++} \mathrm{MCs}$ are referred to as non-classical monocytes which perform a in vivo patrolling function. Mouse MCs are divided into two subsets based on their cell surface expression of Ly6C. The Ly6C high and Ly6C ${ }^{\text {middle }}$ subsets perform pro-inflammatory functions and express high level of CCR2, which is considered the counterpart of human classical MCs. The Ly6 $C^{\text {low }}$ subsets express low level of CCR2, majorly patrol along the vascular endothelium and are involved in tissue repair, functionally similar to human non-classical MCs. CD, cluster of differentiation; CCR2, chemokine (C-C motif) receptor 2 ; $C X 3 C R 1, C X 3 C$ chemokine receptor 1 ; Ly6C, lymphocyte antigen 6 complex.

CX3CR1 via LAF-1/ICAM1-dependent manner. The patrolling behavior of monocytes may be due to low levels expression of adhesion molecules. Ly $6 \mathrm{C}^{-}$monocytes secrete anti-inflammatory cytokine, IL-10 upon in vivo bacterial infection. In vascular inflammation, $\mathrm{Ly}^{-} \mathrm{C}^{-}$ monocytes are recruited to tissue and more likely to differentiate into M2 macrophages, which secrete antiinflammatory cytokine and contribute to tissue repair (Figure 1) [20].
Recruited monocytes/macrophages may emigrate from vessels and enter lymph nodes, which are associated with regression of atherosclerotic lesions [21]. Notably, CD62L (L-selectin) expressed by leukocytes, including $\mathrm{Ly} 6 \mathrm{C}^{+}$ monocytes, is important for circulation to lymph nodes through high endothelial venules (HEV) [15]. Chemokine receptor CCR7 and CCR8, responsible for lymph node traffic, were selectively expressed by Ly6C ${ }^{\text {middle }}$ monocytes [22].

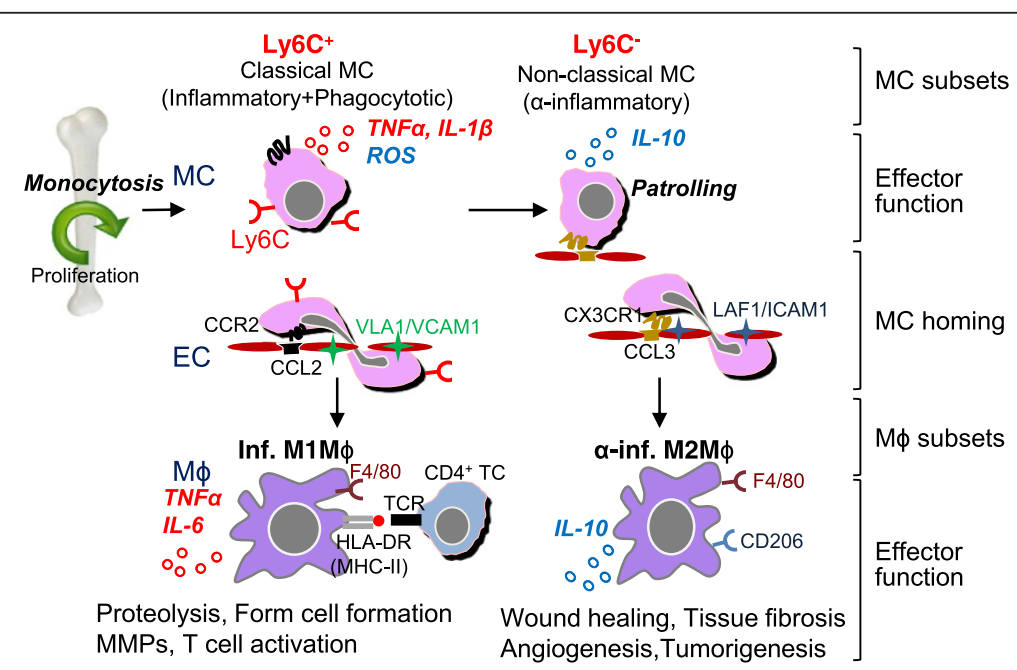

Figure 1 Murine MC and M $\phi$ differentiation, and distinct subset functions. Mouse Ly6C ${ }^{+} \mathrm{MCs}$ leave the bone marrow in a CC-chemokine receptor 2 (CCR2)-dependent manner. In the steady state, Ly6C $C^{+}$MCs differentiate into Ly6C- MCs in the circulation. Ly6C MCs are recruited into normal tissue by interaction of complementary pair CX3CR1/CCL3 via a LAF/ICAM1-dependent manner and become tissue resident M $\phi / D C s$. Ly $6 \mathrm{C}^{+} \mathrm{MCs}$ have a high antimicrobial capability due to their potent capacity for phagocytosis, and secrete ROS, TNFa, and IL-1 $\beta$, whereas Ly6C MCs secrete anti-inflammatory cytokine IL-10 upon in vivo bacteria infection. In vascular inflammation, Ly6C $\mathrm{C}^{+}$MCs are tethered and invade tissue by interaction complimentary pair of CCR2/CCL2(MPC-1) via a VLA-1NCAM1-dependent manner, then mature to inflammatory M1M $\phi$. $\mathrm{M} 1 \mathrm{M} \phi$ are distinguished by secretion of pro-inflammatory cytokines, TNFa and IL-6 and contribute to tissue degradation and T cell activation. Ly6C MCs are recruited to tissue and differentiate into M2M $\phi$, which secrete anti-inflammatory cytokine and contribute to tissue repair. $T C$, $T$ cell; $M C$, monocyte; $M \phi$, macrophage; $E C$, endothelial cells; DC, dendritic cell; inf., inflammatory; a-inf. Anti-inflammatory; TCR, T cell receptor; HLA-DR, human leukocyte antigen DR (a major histocompatibility complex class II (MHC-II)). 


\section{Human monocyte subsets}

Because CD14 is abundantly expressed on the surface of human monocytes and macrophages, it is used to mark human monocytes. Compared to $\mathrm{CD}_{14}{ }^{+} \mathrm{CD} 16^{-}$(also described as $\left.\mathrm{CD} 14^{\text {bright }} \mathrm{CD} 16^{-}\right)$monocytes, the human $\mathrm{CD} 14^{+} \mathrm{CD} 16^{+}$(also described as $\mathrm{CD} 14^{\mathrm{dim}} \mathrm{CD} 16^{+}$) monocyte subset has reduced phagocytic capacity, produces less reactive oxygen species (ROS) and expresses lower levels of CCR2, a chemokine receptor mediating monocyte chemotaxis during inflammation and higher levels of CX3CR1, a chemokine receptor mediating resident monocyte accumulation [23]. Because the chemokine expression pattern implies $\mathrm{CD}^{+} 6^{+}$monocyte has an anti-inflammatory function, there was confusion on the characterization of human monocyte subsets [23]. However, $\mathrm{CD} 14^{+} \mathrm{CD} 16^{+}$monocytes also express CCR2 and are associated with Crohn's disease [24] and CVD [25]. Several earlier clinical studies used $\mathrm{CD} 14^{+} \mathrm{CD} 16^{+}$as the inflammatory monocyte criteria and established the association of increased levels of $\mathrm{CD} 14^{+} \mathrm{CD} 16^{+}$monocyte in human inflammatory diseases, including rheumatoid arthritis, coronary arterial disease, atherosclerosis, hemophagocytic syndrome, and Crohn's disease (Table 3). Moreover, circulating $\mathrm{CD}^{+} 6^{+}$monocyte levels are positively correlated with levels of atherogenic lipids [26] and plaque vulnerability [27], whereas it is negatively correlated with cardiac function such as left ventricular (LV) ejection fraction after AMI [28]. Significant increases in $\mathrm{CD} 6^{+}$monocyte levels have been described in human chronic pathologies in obesity as well [29]. In the same study, several groups reported differential expression of CD $14^{\text {dim }}$ and CD $14^{\text {high }}$ within $\mathrm{CD} 16^{+}$monocytes $[26,30]$, which was related to distinct functional properties of the chemokine receptor expression pattern [31]. A panel of leading experts in monocyte biology proposed consensus nomenclature for human monocyte subsets in 2010, and classified human monocytes subsets as classical monocytes $\left(\mathrm{CD} 14^{++} \mathrm{CD} 16^{-}\right)$, intermediate monocytes $\left(\mathrm{CD} 14^{++} \mathrm{CD} 16^{+}\right)$, and non-classical monocytes $\left(\mathrm{CD} 14^{+} \mathrm{CD} 16^{++}\right)$[32].

As indicated in Table 2, CD $14^{++} \mathrm{CD} 16^{+}$monocytes express CCR2 and selectively CCR5, which react with macrophage inflammatory protein- $1 \alpha$ (MIP-1 $\alpha)$, a chemotactic chemokine for macrophages and CCL5 (termed regulated on activation, normal $\mathrm{T}$ cell expressed and secreted, RANTES). CCR5, known as a co-receptor for human immunodeficiency virus entry into macrophages, is also associated with CVD [31,33]. CD $14^{++} \mathrm{CD} 16^{-}$monocytes express highest levels of CCR2 and $\mathrm{CD} 14^{+} \mathrm{CD} 16^{++}$monocytes express highest levels of CX3CR1 [31].

Although much more evidence supports that $\mathrm{Ly} 6 \mathrm{C}^{+}$and $\mathrm{CD} 14^{+} \mathrm{CD} 16^{-}$classical monocytes are pro-inflammatory monocytes, their high expressions of CD62L imply a possible role of lymph node migration and differentiate into a variety of macrophages and dendritic cell subtypes that could inhibit immune response [34]. Understanding the functions of subsets provides an insight in extrapolating results from clinical studies of inflammatory monocytosis found in patients' blood with various inflammatory diseases.

\section{Functional properties of human monocyte subsets}

As shown in Figure 2, human $\mathrm{CD} 14^{++} \mathrm{CD} 16^{-}$classical monocytes express high levels CCR2 and CD62L (Lselectin), and low levels of CX3CR1. Their major function is phagocytosis. They are phagocytic, exhibit high peroxidase activity, and produce high levels of IL-10 and low levels of TNF- $\alpha$ in response to LPS [23,35]. Gene expression profiling analysis indicates that human classical monocytes preferentially express genes involved in angiogenesis, wound healing, and coagulation [36]. Human $\mathrm{CD}_{14}{ }^{++} \mathrm{CD} 16^{+}$intermediate monocytes display

Table 3 Frequency of two MC subsets in human diseases

\begin{tabular}{|c|c|c|c|c|}
\hline Disease & $\begin{array}{c}\mathrm{CD} 14^{++} \mathrm{CD} 16^{-} \\
\text {(classical, phagocytic) }\end{array}$ & $\begin{array}{c}\mathrm{CD} 14^{+} \mathrm{CD} 16^{+} \\
\text {(Non-classical, inflammatory) }\end{array}$ & $\begin{array}{l}\text { Functional changes } \\
\text { associated with CD14 } \\
\text { CD } 16^{+} \text {MC expansion }\end{array}$ & PMID \# \\
\hline Rheumatoid Arthritis & No change & $2.2 \% \uparrow$ & $\begin{array}{c}\text { HLA-DR and CCR5 } \uparrow \text { Counts } \\
\text { of tender/swollen joints } \uparrow \\
\text { Rheumatoid factors } \uparrow\end{array}$ & 12384915 \\
\hline CAD & & $2.2 \% \uparrow$ & Serum TNFa $\uparrow$ & 15269840 \\
\hline$C A D$ & & $8 \% \uparrow \uparrow$ & Plaque vulnerability $\uparrow$ & 20684824 \\
\hline Atherosclerosis & $8 \% \downarrow$ & $8 \% \uparrow \uparrow$ & & 19461894 \\
\hline Hemophagocytic syndrome & & $31 \%$ & Serum TNFa \& IL-6个 & 17619880 \\
\hline Crohn's disease & & $5.7 \% \uparrow$ & & 17260384 \\
\hline Tumor/haematological malignancy & & $13.3 \% \uparrow$ & & 10209505 \\
\hline
\end{tabular}

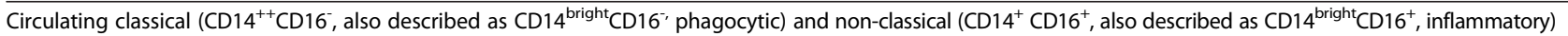
MC counts were examined in human disease as indicated. The percentage change of MC subsets and some functional measurements are recorded. We used PMID \# to cite individual manuscripts reporting these studies. $M C$, monocyte; $A M I$, acute myocardial infarction; $C A D$, coronary arterial disease; $C K D$, chronic kidney disease; $H L A-D R$, human leukocyte antigen DR (MHC-II, major histocompatibility complex class II); TNFa, tumor necrosis factor a; IL-6, interleukin 6; $\uparrow$, increase. 


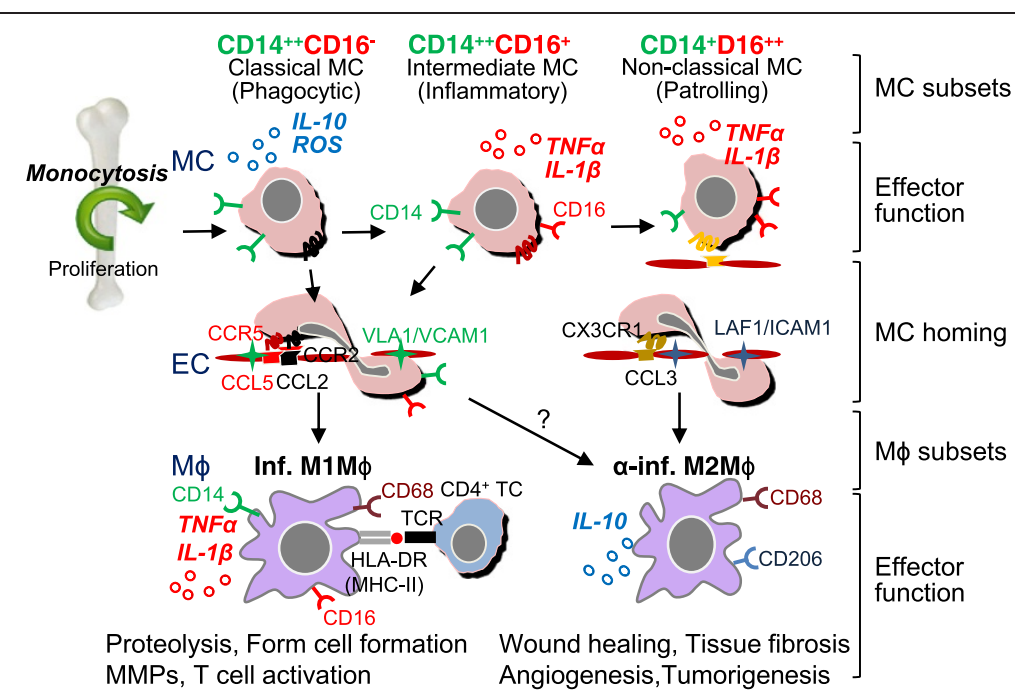

Figure 2 Human $M C$ and $\mathbf{M} \boldsymbol{\phi}$ differentiation, and distinct subset functions. Human $C D 14^{++} C D 16$ classical MCs leave the bone marrow in a CC-chemokine receptor 2 (CCR2)-dependent manner. In the steady state, classical MCs can differentiate into intermediate MCs, then differentiate into patrolling non-classical MCs in circulation. Classical MCs have a high antimicrobial capability due to their potent capacity of phagocytosis, and secrete ROS and IL-10 upon LPS stimulus, whereas intermediate and non-classical MCs secrete inflammatory cytokines, TNFa and IL-1 $\beta$ upon inflammatory stimulation. During inflammation, classical and intermediate MCs are tethered and invade tissue by interaction of complementary pair CCR2/CCL2(MCP1) or/and CCR5/CCL5(RANTES) in a VLA1/NCAM1 dependent manner. MCs then mature to $M 1 M \phi$ in tissue and present self-antigen via MHC-I/II to TCR leading to TC activation. Non-classical MCs patrol the vessel wall and invade by interaction of complementary pair of CX3CR1/CCL3 via LAF/ICAM1-dependent manner. TC, T cell; MC, monocyte; M $\phi$, macrophage; EC, endothelial cells; inf., inflammatory; a-inf. Anti-inflammatory; TCR, T cell receptor; HLA-DR, human leukocyte antigen DR (a major histocompatibility complex class II (MHC-II)).

Table 4 Frequency of three monocyte subsets in different diseases

\begin{tabular}{|c|c|c|c|c|c|}
\hline Disease & $\begin{array}{c}\mathrm{CD} 14^{++} \mathrm{CD} 16^{-} \\
\text {(Classical, phagocytic) }\end{array}$ & $\begin{array}{c}\mathrm{CD} 14^{++} \mathrm{CD} 16^{+} \\
\text {(Intermediate, inflammatory) }\end{array}$ & $\begin{array}{c}\mathrm{CD} 14^{+} \mathrm{CD} 16^{++} \\
\text {(Non-classical, patrolling) }\end{array}$ & $\begin{array}{l}\text { Functional change associated } \\
\text { with } \mathrm{CD} 14^{++} \mathrm{CD} 16^{-} \mathrm{MC} \text { expansion }\end{array}$ & PMID \# \\
\hline Congestive HF & & $6.4 \% \uparrow$ & & $\begin{array}{c}\text { CD143 (ACE) } \uparrow, \text { Creatine } \uparrow, ~ G F R \downarrow, \\
\text { albumin } \downarrow\end{array}$ & 20364047 \\
\hline CKD & & $42 \rightarrow 70$ cells $/ \mu \mathrm{l}$ & 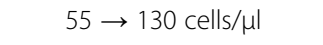 & & 20943670 \\
\hline RA & & $5 \% \uparrow$ & & Th17 cells expansion & 22006178 \\
\hline AAA & & $2.24 \% \uparrow$ & $1.9 \% \uparrow$ & & 23348634 \\
\hline Stroke & & $3 \% \uparrow$ & $3 \% \downarrow$ & & 19293821 \\
\hline HIV-2 & & $7 \% \uparrow$ & & Myeloid dendritic cell depletion & 23460749 \\
\hline Sepsis & No change & $11.5 \% \uparrow$ & $6 \% \uparrow$ & $\begin{array}{c}\text { Phagocytosis } \downarrow \text {, CD86个, HLA-DR } \downarrow \text {, } \\
\text { IL-1 } \beta \downarrow, I L-10 \uparrow\end{array}$ & 12028567 \\
\hline Sepsis & $9.5 \% \downarrow$ & $12 \% \uparrow$ & $3.4 \% \downarrow$ & HLA-DR $\downarrow$, TNFa \& IL-1 $\beta \downarrow, I L-10 \uparrow$ & 19604380 \\
\hline Hepatitis B & $6.2 \% \downarrow$ & $3.3 \% \uparrow$ & $2.5 \% \uparrow$ & $\begin{array}{l}\text { HLA-DR } \uparrow, T N F \text { a } \uparrow, I L-6 \uparrow, I L 1 \beta \uparrow, \\
\text { Th17 cells expansion }\end{array}$ & 21390263 \\
\hline HIV & $2.5 \% \downarrow$ & $3 \% \uparrow$ & $3 \% \uparrow$ & CD163(scavenger receptor)^ & 21625498 \\
\hline Denque fever & $12 \sim 18 \% \downarrow$ & $3 \sim 7 \% \uparrow$ & & 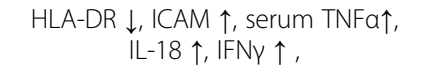 & 20113369 \\
\hline Tuberculosis & $10 \% \downarrow$ & $9 \% \uparrow$ & $13 \% \uparrow$ & TNFa $\uparrow$, apoptosis $\uparrow, \|-10 \downarrow$ & 21621464 \\
\hline
\end{tabular}

Circulating classical (CD14 ${ }^{++} \mathrm{CD} 16^{-}$, also described as CD14 ${ }^{+} \mathrm{CD} 16^{-}$, phagocytic), intermediate $\left(\mathrm{CD} 14^{++} \mathrm{CD} 16^{+}\right.$, also described as $\mathrm{CD} 14^{+} \mathrm{CD}^{+} 6^{+}$, inflammatory) and non-classical $\left(C D 14^{+} \mathrm{CD} 16^{++}\right.$, also described as $\mathrm{CD} 14^{\mathrm{dim}} \mathrm{CD} 16^{+}$, patrolling) MC counts were examined in human disease as indicated. The percentage change of monocyte subsets and some functional measurements are recorded. We used PMID \# to cite individual manuscripts reporting these studies. ACE, angiotensin converting factor; GFR, glomerular filtration rate; CD86, co-stimulatory molecule, HLA-DR, human leukocyte antigen DR (MHC-II, major histocompatibility complex class II); $R A$, rheumatoid arthritis; $A A A$, abdominal aortic aneurysms; $H F$, heart failure; $C K D$, chronic kidney disease; GFR, glomerular filtration rate; $H I V$, human immunodeficiency virus; $\uparrow$, increase; $\downarrow$, decrease; $\rightarrow$, change to. 
inflammatory function. This subset has low peroxidase activity but higher capacity to produce and release IL$1 \beta$, and TNF $\alpha$ in response to LPS [35]. Gene signature links $\mathrm{CD} 14^{++} \mathrm{CD} 16^{+}$monocytes to antigen presentation and $\mathrm{T}$ cell activation (Figure 2) [36]. During inflammation, classical and intermediate monocytes are tethered and invade tissue by interaction of complementary pair of CCR2/CCL2 (termed monocyte chemoattractant protein, MCP) or/and CCR5/CCL5 in a Very Late Activation Antigen-1 (VLA1)/VCAM1 dependent manner.

Human $\mathrm{CD} 14^{+} \mathrm{CD} 16^{++}$non-classical monocytes, patrol the vessel wall and invade by interaction of complementary pair of CX3CR1/CCL3 via the Leu-CAM family integrin lymphocyte functional antigen-1 (LFA-1)/ICA M1-dependent manner (Figure 2). This subset releases IL-1 $\beta$, and TNF $\alpha$ in response to DNA, RNA particles, implicating the pathological role in autoimmune disease such as rheumatoid arthritis [35].

In human CVD and inflammatory conditions, inflammatory intermediate $\mathrm{CD} 14^{++} \mathrm{CD} 16^{+}$monocyate is increased (Tables 3 \& 4). However, the change of $\mathrm{CD} 14^{+}$ $\mathrm{CD}_{16}{ }^{++}$non-classical monocyte count is inconsistent; $\mathrm{CD} 14^{+} \mathrm{CD} 16^{++}$monocyte count is increased in chronic kidney disease (CKD), abdominal aortic aneurysms (AAA), sepsis, hepatitis B, human immunodeficiency virus (HIV) infection and tuberculosis, but decreased in congestive heart failure, stroke and sepsis. It was suggested that $\mathrm{CD} 14^{++} \mathrm{CD} 16^{-}$and $\mathrm{CD} 14^{++} \mathrm{CD} 16^{+}$monocytes resemble mouse $\mathrm{Ly} 6 \mathrm{C}^{+}$inflammatory monocyte subset, whereas $\mathrm{CD} 14^{+} \mathrm{CD} 16^{++}$monocytes may resemble Ly6C anti-inflammatory monocytes and have potential role of patrolling vascular endothelium [23]. However, some studies emphasize the inflammatory role of $\mathrm{CD} 14^{+} \mathrm{CD} 16^{++}$ cells because of the production of inflammatory cytokines. Nevertheless, much attention has been focused on the changes of $\mathrm{CD} 14^{++} \mathrm{CD} 16^{+}$intermediate monocyte count in patients with inflammatory diseases. Since $\mathrm{CD} 14^{++} \mathrm{CD} 16^{+}$monocyte count increases are consistently associated with human inflammatory disease (Tables 3 \& 4), it is a sufficient biomarker of chronic and acute inflammatory diseases.

\section{Monocyte differentiation}

Monocytes are differentiated from the committed precursor termed macrophage-DC precursor (MDP) mainly resident in bone marrow and differentiate into either dendritic cells or macrophages. They consist of two main subpopulations: CX3CR $1^{\text {high }} \mathrm{CCR} 22^{\text {low }} \mathrm{Ly} 6 \mathrm{C}^{-}$and CX3CR $1{ }^{\text {low }} \mathrm{CCR} 2^{\text {high- }}$ Ly6C $\mathrm{C}^{+}$. However, it is unclear whether $\mathrm{Ly}^{-} \mathrm{C}^{-}$monocyte is differentiated from CX3CR $1{ }^{\text {low }} \mathrm{CCR} 2{ }^{\text {high }} \mathrm{Ly} 6 \mathrm{C}^{+}$or directly from bone marrow MDP. After maturation, $\mathrm{Ly} 6 \mathrm{C}^{+}$monocytes leave bone marrow and enter into the blood stream via CCR2 mediated migration [37]. After leaving the bone marrow, mouse $\mathrm{Ly}_{6 \mathrm{C}^{+}}$monocytes differentiate into Ly6C monocytes in circulation [38]. A recent monocyte fate mapping study strongly supported that in the steady state, Ly6C $\mathrm{C}^{+}$monocyte is the obligatory precursor for generation and lifespan control of Ly6 $\mathrm{C}^{-}$monocyte in the bone marrow, peripheral blood and spleen. In a competitive setting of mixed CCR2-proficient (CD45.1) and CCR2deficient (CD45.2) (Ly6C $\mathrm{C}^{+}$monocytes are reported to be selectively reduced) $\mathrm{BM}$ chimeras, $\mathrm{CD}_{45.1^{+}} \mathrm{WT} \mathrm{Ly} 6 \mathrm{C}^{-}$ monocytes outcompeted their $\mathrm{CD} 45.2$ mutant $\mathrm{Ly} 6 \mathrm{C}^{-}$counterparts [39]. In the same study, $\mathrm{Ly}_{6} \mathrm{C}^{+}$monocytes restored regained Ly6 $\mathrm{C}^{-}$half-life and the population.

Similarly, in human monocyte differentiation, it is accepted that $\mathrm{CD} 14^{++}$classical monocytes leave bone marrow and differentiate into $\mathrm{CD} 14^{++} \mathrm{CD} 16^{+}$intermediate monocytes and sequentially to $\mathrm{CD} 14^{+} \mathrm{CD} 16^{++}$nonclassical monocytes in peripheral blood circulation [40].

\section{Monocyte to macrophage differentiation}

$\mathrm{CCR} 2{ }^{\text {hi }}$ Ly $6 \mathrm{C}^{+}$inflammatory and $\mathrm{CCR}^{\text {low }}{ }^{2} 6 \mathrm{C}^{-}$resident monocytes are generally thought to preferentially differentiate into M1 inflammatory and M2 antiinflammatory macrophages, respectively, during early inflammation [20]. $\mathrm{Ly}_{6} \mathrm{C}^{+}$monocytes dominate the early phase of myocardial infarction and exhibit phagocytic, proteolytic, inflammatory function and digest damaged tissue. On the other hands, Ly6 $\mathrm{C}^{-}$monocytes, recruited at later phase of inflammation, attenuate inflammatory properties and differentiate toward M2 macrophages and contribute to angiogenesis, genesis of my fibroblasts, and collagen deposition (Figure 1). It is possible that monocytes and macropahge are highly plastic and can be crossly differentiated into different subsets in response to environment changes. Several studies revealed "unusual" cascades of monocytes to macrophage transition: 1) Infiltrated $\mathrm{Ly}_{6} \mathrm{C}^{+}$monocytes in inflamed skeletal muscle or brain tissues acquire phenotypic features of anti-inflammatory monocytes by down-regulating Ly6C expression, thereby displaying anti-inflammatory M2 macrophages function; [41,42] 2) Ly $6 \mathrm{C}^{\text {middle }}$ monocytes emigrate to lymph nodes via CCR7 and CCR8 and differentiate into dendritic cells; $[22,43] 3)$ During steady state, Ly6C ${ }^{+}$monocytes are recruited to healthy lamina propria and differentiate into tissue resident CX3CR1 $1^{\text {high }}$ macrophages; [44] 4) M2 macrophages are generated by alternative activation of tissue-resident macrophages rather than recruited monocytes during infection with Litomosoides sigmodontis; [45] and 5) Inflammatory monocyte recruitment to allergic skin is essential to alleviate allergic inflammation in order to acquire an anti-inflammatory M2 phenotype via basophil-derived IL-4 [46]. These findings demonstrated the multiple capacities of monocytes to differentiate into either regulatory or inflammatory mature macrophages/dendritic cells. 


\section{Inflammatory monocytosis in CVD and stroke}

Inflammatory monocytes are the major cellular component in atherosclerotic plaque [47]. Accumulation of activated immune cells, including inflammatory monocytes and macrophages, and $\mathrm{T}$ lymphocytes in the vessel wall produce inflammatory cytokines and facilitate vascular inflammation.

Inflammatory monocytes may contribute to vascular inflammation not only by producing inflammatory cytokines, but also via CD40-mediated $\mathrm{T}$ cell activation. It was reported that $\mathrm{CD} 40-\mathrm{CD} 40$ ligand $(\mathrm{CD} 40 \mathrm{~L})$ signaling, a $\mathrm{T}$ cell co-stimulatory receptor-ligand pair, plays a crucial role in atherosclerosis [48]. The action of $\mathrm{T}$ cells in atherosclerosis is similar to a $\mathrm{CD} 4^{+} \mathrm{T}$ helper cell 1 (Th1)-mediated hypersensitivity reaction, which might use ox-LDL as a possible auto-antigenic stimulus [49]. In human atherosclerotic lesions, CD40-CD40L are co-localized with epitopes of ox-LDL, scavenger receptor A (a mediator of foam cell formation), and CD16 [50]. CD40 is a TNF receptor superfamily 5 member and is expressed in monocytes, macrophages, dendritic cells. CD40 ligand is found on $\mathrm{CD} 4^{+} \mathrm{T}$ cells and platelets in both secreted and membrane bound forms. CD40-CD40L expression on platelets enhances platelet activation and thrombosis [51]. CD40 and CD40L are both expressed on endothelial cells and vascular smooth muscle cells. Either CD40 or CD40L deficiency in $\mathrm{ApoE}^{-/-}$mice abrogated atherosclerosis by increasing the extracellular matrix and promoting M2 macrophage polarization [52].

Classical $\mathrm{CD}_{1} 4^{+}$monocytes are critical for clearance of $\mathrm{LDL}$, whereas $\mathrm{CD} 16^{+}$monocytes including intermediate and nonclassical monocytes have higher expression levels of major histocompatibility complex class II (MHC-II) and higher capacity to uptake ox-LDL [53]. CD40 signaling induced the expression of adhesion molecules, matrix metalloproteinases and proinflammatory cytokines in macrophages and foam cell formation [54]. It was reported that monoclonal antibodies against CD40L reduced atherosclerosis rendered thromboembolic complications [55]. Thus, antagonizing CD40 signaling or suppressing $\mathrm{CD} 40$ expression might be future therapeutic alternatives for human CVD.

Similarly, monocytes are the major infiltrating immune cells in the ischemic brain in stroke. Monocyte infiltration is one of the earliest cellular response in stroke. It occurs 4 hours after stroke and reaches maximum infiltration in 7 days [56]. Inflammation accompanying stroke plays an important role in secondary ischemic injury [57]. Infiltrated inflammatory cells can produce ROS, inflammatory cytokines and matrix metalloproteinase, inducing neuron injury directly or indirectly by inducing blood brain barrier (BBB) disruption, which can lead to edema, cerebral hemorrhage and a vicious circle of continuous influx of myeloid cells. However, the inflammatory effects on the stroke process can be detrimental or protective, depending on the immune cell types, numbers and duration. A recently published paper indirectly supported the detrimental role of monocytes in stroke [58]. Bone marrow transplantation from $\mathrm{ApoE}^{-/-} \mathrm{CD} \mathrm{6}^{-1-}$ (mostly expressed in monocytes) donor mice to $A p o E^{-1-}$ recipient mice decreased infarction volume and neurological deficits after stroke. But the roles of different monocyte subsets in the pathogenesis of stroke remain unclear. Ly $6 \mathrm{C}^{+}$monocytes have been proven to be responsible for many central nervous system diseases like autoimmune multiple sclerosis [59] and infectious encephalitis caused by West Nile virus [60]. The chemokine receptor CCR2 deficiency, which is the main chemokine receptor for recruiting $\mathrm{Ly}_{6} \mathrm{C}^{+}$monocytes, attenuates infarction size and neurological deficit after stroke in the transient middle cerebral artery occlusion (tMCAO) stroke mouse model, accompanying significantly reduced monocyte and neutrophil infiltration [61]. Also, there is a report pointing out that the $\mathrm{Ly}_{6} \mathrm{C}^{-}$ macrophages differentiated from infiltrating $\mathrm{Ly} 6 \mathrm{C}^{+}$ monocytes are critical for preventing hemorrhagic infarct transformation in both the $\mathrm{tMCAO}$ and the photo thrombosis induced permanent stroke models [62]. However, Ly $6 \mathrm{C}^{+}$monocyte depletion by clondronate liposome or by bone marrow transplantation from $C C R 2^{-/-}$donor mice to wild type recipient mice showed dramatically increased hemorrhage occurrence rates without changing infarction volume and neurological function. The reason why the same CCR2 deficiency mice display different results is unknown, it may be due to different mouse breeding methods since pure knockout mouse cross-breeding for several generations may lead to gene changes, which may compensate for the designated gene defect. To determine the roles of different monocyte subsets in stroke pathogenesis, more experiments should be conducted in the context of normal or combined disease settings like hyperlipidemia and hyperhomocysteinemia.

\section{Conclusion}

To date, many studies have demonstrated the key roles of inflammatory and anti-inflammatory monocytes in response to inflammation or steady state in mouse models. Inflammatory monocyte subset is a valuable biomarker for human inflammatory diseases, including cardiovascular diseases. Understanding the mechanism of monocyte differentiation will likely provide a potential therapeutic target for inflammatory monocytosis.

\section{Competing interests}

The authors declare that they have no competing interests.

\section{Authors' contributions}

YJ generated figures and tables and drafted manuscript. ZL contributed on text for inflammatory monocytosis in stroke and partially monocyte

differentiation. YC contributed on scientific discussion and editing scientific 
writing and English. X-FY contributed to manuscript design and final review. $\mathrm{WH}$ is responsible for the manuscript design and final writing. All authors read and approved the final manuscript.

\section{Acknowledgements}

This work was supported in part by NIH Grants HL67033, HL77288, HL82774, HL110764 and HL117654 (HW); and HL9445, HL108910 and HL116917 (XFY).

Received: 23 November 2013 Accepted: 20 December 2013 Published: 7 January 2014

\section{References}

1. Yasaka T, Mantich NM, Boxer LA, Baehner RL: Functions of human monocyte and lymphocyte subsets obtained by countercurrent centrifugal elutriation: differing functional capacities of human monocyte subsets. J Immunol 1981, 127:1515-1518.

2. Kurihara T, Warr G, Loy J, Bravo R: Defects in macrophage recruitment and host defense in mice lacking the CCR2 chemokine receptor. J Exp Med 1997, 186:1757-1762.

3. Nahrendorf $M$, et al: The healing myocardium sequentially mobilizes two monocyte subsets with divergent and complementary functions. J Exp Med 2007, 204:3037-3047. 10.1084/jem.20070885.

4. Ingersoll MA, Platt AM, Potteaux S, Randolph GJ: Monocyte trafficking in acute and chronic inflammation. Trends Immunol 2011, 32:470-477. 10.1016/j.it.2011.05.001

5. Meuret G, Bammert J, Hoffmann G: Kinetics of human monocytopoiesis. Blood 1974, 44:801-816.

6. Afiune Neto A, Mansur Ade P, Avakian SD, Gomes EP, Ramires JA: [Monocytosis is an independent risk marker for coronary artery disease]. Arquivos brasileiros de cardiologia 2006, 86:240-244. S0066-782X2006000300013.

7. Maekawa $Y$, et al: Prognostic significance of peripheral monocytosis after reperfused acute myocardial infarction: a possible role for left ventricular remodeling. J Am Coll Cardiol 2002, 39:241-246.

8. Ganda A, et al: Mild Renal Dysfunction and Metabolites Tied to Low HDL Cholesterol Are Associated With Monocytosis and Atherosclerosis. Circulation 2013, 127:988-996. 10.1161/CIRCULATIONAHA.112.000682.

9. Chapman CM, Beilby JP, McQuillan BM, Thompson PL, Hung J: Monocyte count, but not C-reactive protein or interleukin-6, is an independent risk marker for subclinical carotid atherosclerosis. Stroke 2004, 35:1619-1624. 10.1161/01.STR.0000130857.19423.ad.

10. Tani S, et al: Association of leukocyte subtype counts with coronary atherosclerotic regression following pravastatin treatment. Am J Cardiol 2009, 104:464-469. 10.1016/j.amjcard.2009.04.009.

11. Hurst NP, Nuki G: Evidence for defect of complement-mediated phagocytosis by monocytes from patients with rheumatoid arthritis and cutaneous vasculitis. Br Med J (Clin Res Ed) 1981, 282:2081-2083.

12. Schechter GP, Wahl LM, Oppenheim JJ: Suppressor monocytes in human disease: a review. Adv Exp Med Biol 1979, 121B:283-298.

13. Clarkson SB, Ory PA: CD16. Developmentally regulated IgG Fc receptors on cultured human monocytes. J Exp Med 1988, 167:408-420.

14. Passlick B, Flieger D, Ziegler-Heitbrock HW: Identification and characterization of a novel monocyte subpopulation in human peripheral blood. Blood 1989, 74:2527-2534.

15. Palframan RT, et al: Inflammatory chemokine transport and presentation in HEV: a remote control mechanism for monocyte recruitment to lymph nodes in inflamed tissues. J Exp Med 2001, 194:1361-1373.

16. Si Y, Tsou CL, Croft K, Charo IF: CCR2 mediates hematopoietic stem and progenitor cell trafficking to sites of inflammation in mice. $J$ Clin Investig, 120:1192-1203.

17. Serbina NV, Jia T, Hohl TM, Pamer EG: Monocyte-mediated defense against microbial pathogens. Annu Rev Immunol 2008, 26:421-452. 10.1146/annurev.immunol.26.021607.090326.

18. Barbalat R, Lau L, Locksley RM, Barton GM: Toll-like receptor 2 on inflammatory monocytes induces type I interferon in response to viral but not bacterial ligands. Nat Immunol 2009, 10:1200-1207. 10.1038/ni.1792

19. Audoy-Remus J, et al: Rod-Shaped monocytes patrol the brain vasculature and give rise to perivascular macrophages under the influence of proinflammatory cytokines and angiopoietin- 2 . J Neurosci 2008, 28:10187-10199. 10.1523/JNEUROSCI.3510-08.2008.
20. Auffray $C$, et al: Monitoring of blood vessels and tissues by a population of monocytes with patrolling behavior. Science 2007, 317:666-670. 10.1126/science.1142883

21. Randolph GJ: Emigration of monocyte-derived cells to lymph nodes during resolution of inflammation and its failure in atherosclerosis. Curr Opin Lipidol 2008, 19:462-468. 10.1097/MOL.0b013e32830d5f09.

22. Qu C, et al: Role of CCR8 and other chemokine pathways in the migration of monocyte-derived dendritic cells to lymph nodes. J Exp Med 2004, 200:1231-124. 10.1084/jem.20032152.

23. Geissmann F, Jung S, Littman DR: Blood monocytes consist of two principal subsets with distinct migratory properties. Immunity 2003, 19:71-82.

24. Grip O, Bredberg A, Lindgren S, Henriksson G: Increased subpopulations of CD16(+) and CD56(+) blood monocytes in patients with active Crohn's disease. Inflamm Bowel Dis 2007, 13:566-572. 10.1002/ibd.20025.

25. Shantsila $E$, et al: Immunophenotypic characterization of human monocyte subsets: possible implications for cardiovascular disease pathophysiology. J Thromb Haemost 2011, 9:1056-1066. 10.1111/j.1538-7836.2011.04244.x.

26. Rothe $\mathrm{G}$, et al: Peripheral blood mononuclear phagocyte subpopulations as cellular markers in hypercholesterolemia. Arterioscler Thromb Vasc Biol 1996, 16:1437-1447.

27. Kashiwagi $M$, et al: Association of monocyte subsets with vulnerability characteristics of coronary plaques as assessed by 64-slice multidetector computed tomography in patients with stable angina pectoris. Atherosclerosis 2010, 212:171-176. 10.1016/j.atherosclerosis.2010.05.004.

28. Tsujioka $\mathrm{H}$, et al: Impact of heterogeneity of human peripheral blood monocyte subsets on myocardial salvage in patients with primary acute myocardial infarction. J Am Coll Cardiol 2009, 54:130-138. 10.1016/j. jacc.2009.04.021.

29. Rogacev KS, et al: Monocyte heterogeneity in obesity and subclinical atherosclerosis. Eur Heart J 2010, 31:369-376. 10.1093/eurheartj/ehp308.

30. Tanaka $M$, et al: Surface phenotype analysis of CD16+ monocytes from leukapheresis collections for peripheral blood progenitors. Clin Exp Immunol 1999, 116:57-61.

31. Ancuta $P$, et al: Fractalkine preferentially mediates arrest and migration of CD16+ monocytes. J Exp Med 2003, 197:1701-1707.

32. Ziegler-Heitbrock $L$, et al: Nomenclature of monocytes and dendritic cells in blood. Blood 2010, 116:e74-80. 10.1182/blood-2010-02-258558.

33. Jones KL, Maguire JJ, Davenport AP: Chemokine receptor CCR5: from AIDS to atherosclerosis. Br J pharmacol 2011, 162:1453-1469. 10.1111/j.1476-5381.2010.01147.x.

34. Xu H, Manivannan A, Crane I, Dawson R, Liversidge J: Critical but divergent roles for CD62L and CD44 in directing blood monocyte trafficking in vivo during inflammation. Blood 2008, 112:1166-1174. 10.1182/blood-2007-06-098327.

35. Cros J, et al: Human CD14dim monocytes patrol and sense nucleic acids and viruses via TLR7 and TLR8 receptors. Immunity 2010, 33:375-386. 10.1016/.j.immuni.2010.08.012.

36. Wong $\mathrm{KL}$, et al: Gene expression profiling reveals the defining features of the classical, intermediate, and nonclassical human monocyte subsets. Blood 2011, 118:e16-e31. 10.1182/blood-2010-12-326355.

37. Sunderkotter $C$, et al: Subpopulations of mouse blood monocytes differ i n maturation stage and inflammatory response. J Immunol 2004, 172:4410-4417.

38. Hristov M, Weber C: Differential role of monocyte subsets in atherosclerosis. Thromb Haemost 2011, 106:757-762. 10.1160/TH11-07-0500.

39. Yona $S$, et al: Fate mapping reveals origins and dynamics of monocytes and tissue macrophages under homeostasis. Immunity 2013, 38:79-91.

40. Zawada AM, et al: Monocyte heterogeneity in human cardiovascular disease. Immunobiology 2012, 217:1273-1284. 10.1016/j.imbio.2012.07.001.

41. Arnold L, et al: Inflammatory monocytes recruited after skeletal muscle injury switch into antiinflammatory macrophages to support myogenesis. J Exp Med 2007, 204:1057-1069. 10.1084/jem.20070075.

42. Saederup $N$, et al: Selective chemokine receptor usage by central nervous system myeloid cells in CCR2-red fluorescent protein knock-in mice. Plos one 2010, 5:e13693.

43. Zigmond $\mathrm{E}$, et al: Ly6C hi monocytes in the inflamed colon give rise to proinflammatory effector cells and migratory antigen-presenting cells. Immunity 2012, 37:1076-1090. 10.1016/j.immuni.2012.08.026.

44. Rivollier A, He J, Kole A, Valatas V, Kelsall BL: Inflammation switches the differentiation program of Ly6Chi monocytes from antiinflammatory 
macrophages to inflammatory dendritic cells in the colon. J Exp Med 2012, 209:139-155. 10.1084/jem.20101387.

45. Jenkins SJ, et al: Local macrophage proliferation, rather than recruitment from the blood, is a signature of TH2 inflammation. Science 2011, 332:1284-1288. 10.1126/science.1204351.

46. Egawa M, et al: Inflammatory Monocytes Recruited to Allergic Skin Acquire an Anti-inflammatory M2 Phenotype via Basophil-Derived Interleukin-4. Immunity 2013, 38(3):570-580. 10.1016/.jimmuni.2012.11.014.

47. Zhang $D$, et al: Severe hyperhomocysteinemia promotes bone marrowderived and resident inflammatory monocyte differentiation and atherosclerosis in LDLr/CBS-deficient mice. Circ Res 2012, 111:37-49. 10.1161/CIRCRESAHA.112.269472.

48. Mach F, Schonbeck U, Sukhova GK, Atkinson E, Libby P: Reduction of atherosclerosis in mice by inhibition of CD40 signalling. Nature 1998, 394:200-203. 10.1038/28204.

49. Tedgui A, Bernard C: Cytokines, immuno-inflammatory response and atherosclerosis. Eur Cytokine Netw 1994, 5:263-270.

50. Hakkinen T, Karkola K, Yla-Herttuala S: Macrophages, smooth muscle cells, endothelial cells, and T-cells express CD40 and CD40L in fatty streaks and more advanced human atherosclerotic lesions. Colocalization with epitopes of oxidized low-density lipoprotein, scavenger receptor, and CD16 (Fc gammaRIII). Virchows Archiv 2000, 437:396-405.

51. Lievens $D$, et al: Platelet CD40L mediates thrombotic and inflammatory processes in atherosclerosis. Blood 2010, 116:4317-4327. 10.1182/blood2010-01-261206.

52. Lutgens $\mathrm{E}$, et al: Deficient CD40-TRAF6 signaling in leukocytes prevents atherosclerosis by skewing the immune response toward an antiinflammatory profile. J Exp Med 2010, 207:391-404. 10.1084/jem.20091293.

53. Mosig S, et al: Different functions of monocyte subsets in familial hypercholesterolemia: potential function of CD14+ CD16+ monocytes in detoxification of oxidized LDL. FASEB J 2009, 23:866-874. 10.1096/fj.08118240.

54. Phipps RP: Atherosclerosis: the emerging role of inflammation and the CD40-CD40 ligand system. Proc Natl Acad Sci U S A 2000, 97:6930-6932.

55. Kawai T, Andrews D, Colvin RB, Sachs DH, Cosimi AB: Thromboembolic complications after treatment with monoclonal antibody against CD40 ligand. Nat Med 2000, 6:114. 10.1038/72162.

56. Gelderblom M, et al: Temporal and spatial dynamics of cerebral immune cell accumulation in stroke. Stroke 2009, 40:1849-1857.

57. Jin R, Yang G, Li G: Inflammatory mechanisms in ischemic stroke: role of inflammatory cells. J Leukoc Biol 2010, 87:779-789.

58. Kim $E$, et al: $C D 36$ in the periphery and brain synergizes in stroke injury in hyperlipidemia. Ann Neurol 2012, 71:753-764.

59. King IL, Dickendesher TL, Segal BM: Circulating Ly- $6 C+$ myeloid precursors migrate to the CNS and play a pathogenic role during autoimmune demyelinating disease. Blood 2009, 113:3190-3197.

60. Getts DR, et al: Ly6c + "inflammatory monocytes" are microglial precursors recruited in a pathogenic manner in West Nile virus encephalitis. J Exp Med 2008, 205:2319-2337.

61. Dimitrijevic OB, Stamatovic SM, Keep RF, Andjelkovic AV: Absence of the chemokine receptor CCR2 protects against cerebral ischemia/reperfusion injury in mice. Stroke 2007, 38:1345-1353.

62. Gliem $\mathrm{M}$, et al: Macrophages prevent hemorrhagic infarct transformation in murine stroke models. Ann Neurol 2012, 71:743-752.

\section{Submit your next manuscript to BioMed Central and take full advantage of:}

- Convenient online submission

- Thorough peer review

- No space constraints or color figure charges

- Immediate publication on acceptance

- Inclusion in PubMed, CAS, Scopus and Google Scholar

- Research which is freely available for redistribution 